\title{
Modal decomposition of magnetic maps: the case of Cape Roberts aeromagnetic survey, Antarctica
}

\author{
Marco Gambetta \\ Istituto Nazionale di Geofisica e Vulcanologia, Roma, Italy
}

\begin{abstract}
This paper proposes a digital enhancement tool for magnetic anomaly maps. The magnetic anomaly map is decomposed by means of Eigenvalue Decomposition into a number of orthogonal bases. The dataset is then filtered accordingly to a specific variance pattern. The dataset is decomposed and the eigenvalues population is inspected so that the variance is evaluated yielding the definition of two thresholds. Subsequently, the dataset is reconstructed into three subsets which hold different features respectively. The proposed filtering procedure is first tested with a synthetic signal and then applied to the data of Cape Roberts (Antarctica) aeromagnetic survey, flown over an off-shore rift basin. The proposed method appears to be efficient in noise removal and acts as a digital enhancement tool which provides TMI anomaly maps revealing hidden lineaments, otherwise not visible. The methodology effectiveness as a hidden lineaments detection tool has been checked against independent data.
\end{abstract}

Key words Magnetic mapping - Digital Enhancement - Eigenvalues - HRAM - Antarctic Magnetic Surveys

\section{Introduction}

Eigenvalue decomposition is a computational technique widely used in many geophysical fields such as oceanographic (Fukumori and Wunsch, 1991) seismic (Freire and Ulrych, 1988) deep electromagnetic soundings (Egbert and Booker, 1989) and also in image processing (Prasantha et al., 2007). In the last few decades this technique has been extensively used in image coding and compression applied to image transmission over a nationwide computer net-

Mailing address: Dr. Marco Gambetta, Istituto Nazionale di Geofisica e Vulcanologia, Via di Vigna Murata 605, 00153 Roma, Italy; e-mail: marco.gambetta@ingv.it work. This work explores the feasibility of eigenvalue decomposition as a separation tool between signal and noise, used with magnetic maps.

Frequently, subtle magnetic features and lineaments may be concealed both by broad high amplitude anomalies and by residual noise. Such features, like magnetic lineaments caused by intrasedimentary faulting, play a key role in the interpretation of the tectonic setting. The proposed technique is useful in highlighting these kinds of features.

In detail, the magnetic image is decomposed and then represented into a number of orthogonal bases thereby obtaining a new image formed by fewer dimensions. By means of this technique it is possible to separate the meaningful signal from the noise. This decomposition seems to be efficient in noise removal and acts as an enhancement technique able to highlight hidden features. Due to the intrinsic implementation simplicity the procedure can also be used as an interactive tool. 


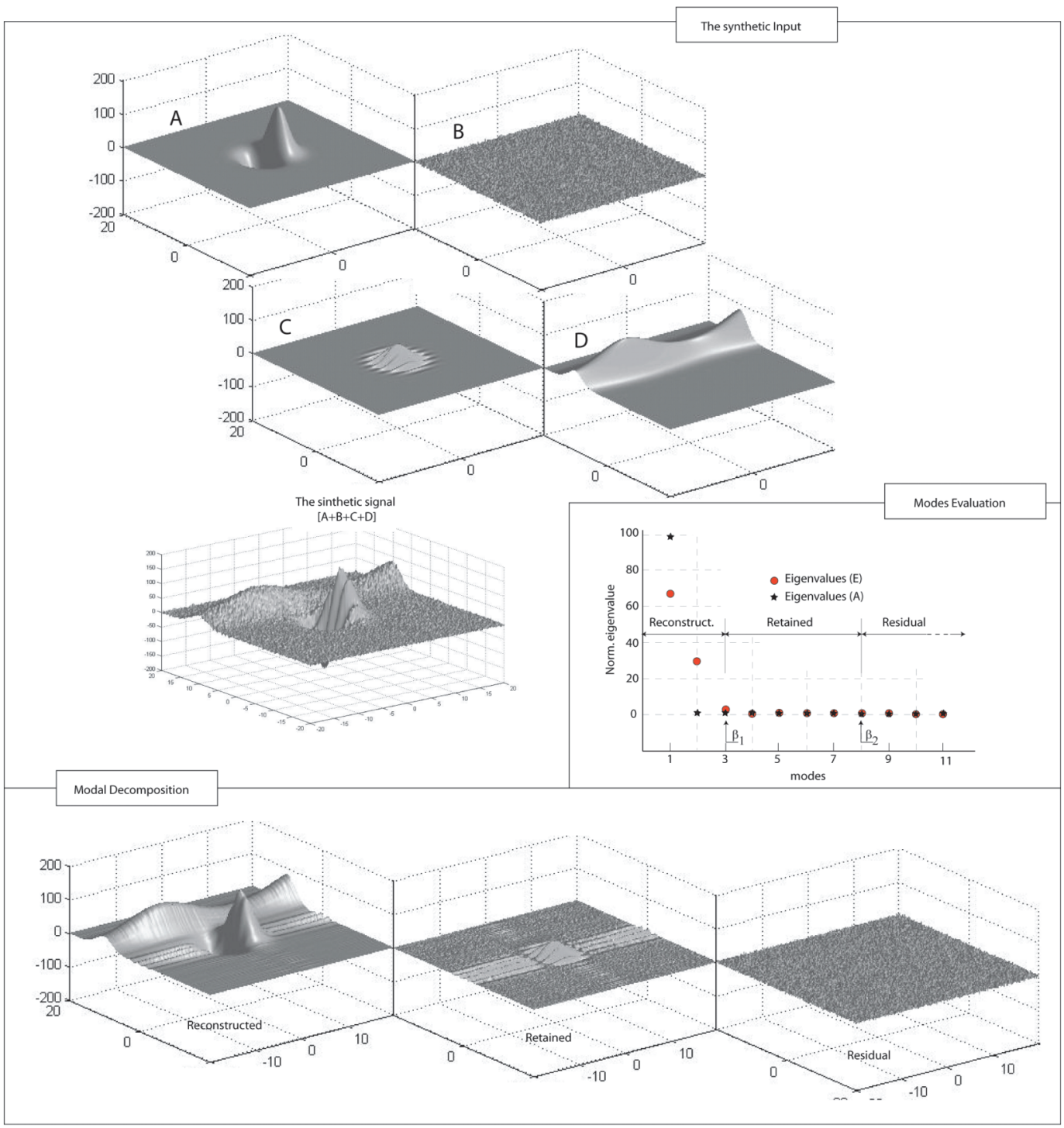

Fig. 1. Test of the modal decomposition with a synthetic model as input.

It should be emphasized that the proposed method is essentially empirical, so the definition of filtering thresholds has to be fixed inspecting the filtering results. As a rule of thumb, large amplitude features are often confined in the lower modes, and because of its low amplitude the noise is confined in the upper modes. The sorted set of eigenvalues is thus a kind of representation of the variance distribution of the magnetic anomaly image, which can be used to separate different kind of data and data from noise. 


\section{Eigenvalue decomposition}

Let $X$ be a $[m \times n]$ zero mean matrix as

$$
X=\left[\begin{array}{l}
x_{1} \\
\vdots \\
x_{m}
\end{array}\right]
$$

where $x_{\mathrm{i}}$ is the $[1 \mathrm{x} n]$ row vector of the magnetic anomaly image.

The covariance matrix of $X$ is

$$
C_{x}=\frac{1}{n-1} X X^{T}
$$

Let us compute the eigenvalues and the eigenvectors of the covariance matrix $C_{x}$

$$
C_{x}=V \Lambda V^{T}
$$

and sort the eigenvectors $V$ in decreasing order by eigenvalues $\Lambda$

Analyzing the behavior of the sorted set of $\Lambda_{i i}$ a general trend is often recognizable. In this paper I propose a filtering technique which acting on $\Lambda_{i i}$ elements provides three filtered dataset.

Let us define two thresholds $\beta_{1}$ and $\beta_{2}$ as follows

$$
\begin{aligned}
& \Lambda_{c}=\left\{\begin{array}{l}
\Lambda_{i i}: i<\beta_{1} \\
0: \geq \beta_{1}
\end{array}\right\} ; \Lambda_{t}=\left\{\begin{array}{l}
0: i \geq \beta_{1} \\
\Lambda_{i i}: \beta_{1} \leq i \leq \beta_{2} \\
0: i \geq \beta_{1}
\end{array}\right\} ; \\
& \Lambda_{r}=\left\{\begin{array}{l}
0: i \leq \beta_{2} \\
\Lambda_{i i}: i>\beta_{2}
\end{array}\right\}
\end{aligned}
$$

with $1<\beta_{1}<\beta_{2}<m$

then let three sets of $V$ named $V_{\text {filt }}$ neglecting those eigenvectors corresponding to a null element of $\Lambda_{i i}$ and regain the transposed filtered data $\left(X_{\text {filt }}\right)$ by:

$$
X_{\text {filt }}=V_{\text {filt }} V_{\text {filt }}^{T} X^{T}
$$

$X_{\text {filt }}$ and $V_{\text {filt }}$ may be named according to the convention used above: $X_{c}$ will hold the «reconstructed» dataset, $X_{t}$ the «retained» dataset and $X_{r}$ the «residual» dataset.

\section{Application to a synthetic case}

In order to check the performance of the decomposition, a square 200x200 pixel image matrix was created. The input signal (fig. 1, upper box) is composed of a large (mean $\mu=0$; peak-to-peak $=270$, arbitrary units) perturbated dipolar anomaly $(\mathrm{A}+\mathrm{C})$, an elongated feature (D), which may mimic some kind of leveling error and a normal random noise $(\mu=0 ; \sigma=3)$. Sketches A to D figure out the components of the compound synthetic signal (E). The box labeled «Modal evaluation» reports a set of eigenvalues calculated using eq. 2.1 to 2.3.

The red circles refer to eigenvalues calculated using the synthetic signal (E) as input; the black stars refer to eigenvalues calculated using the noise-free not perturbated dipolar anomaly (A) as input.

The features B, C, D, forming the synthetic signal $\mathrm{E}$ are visible in the modes from 2 to 4 . The eigenvalues in this plot have been normalized as

$$
\lambda_{i}^{*}=\frac{\Lambda_{i i}}{\sum_{i \rightarrow 1: n} \Lambda_{i i}} \times 100
$$

The eigenvalues plot also shows the two thresholds, $\beta_{1}, \beta_{2}$ used to accomplish the modal decomposition. The bottom box of fig. 1 reports the results of the modal decomposition of the synthetic signal (E) using $\beta_{1}=3, \beta_{2}=9$. The reconstructed signal holds the main features $(\mathrm{A}+\mathrm{D})$, the high frequency perturbation $(\mathrm{C})$ was separated and confined in the retained dataset. The incoherent noise (B) was separated in the residual dataset.

\section{Application to the Cape Roberts Aeromagnetic Survey}

In order to test the proposed method the dataset of the HRAM survey performed in the Cape Roberts area (Victoria Land, Antarctica) (Bozzo et al., 1997) was used. Cape Roberts is a key area to address in further detail the geometry and kinematics of strike-slip faulting and its control on Cenozoic magmatism at the boundary between the Transantarctic Moun- 


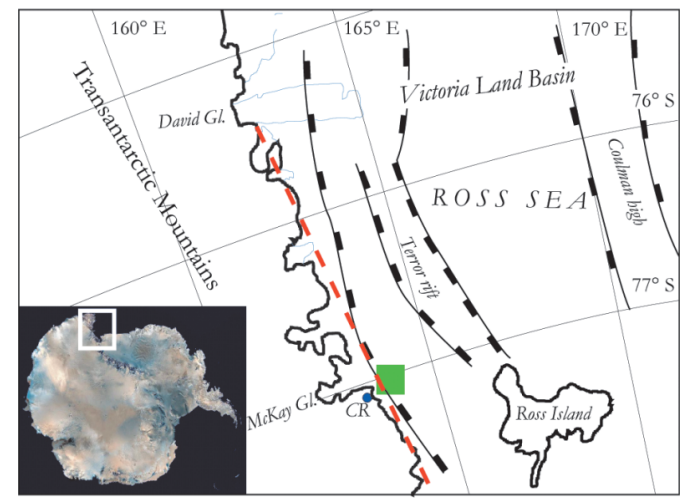

Fig. 2. Location of Cape Roberts area. Red dashed line: McMurdo sound fault system, simplified from (Ferraccioli et al., 2003).

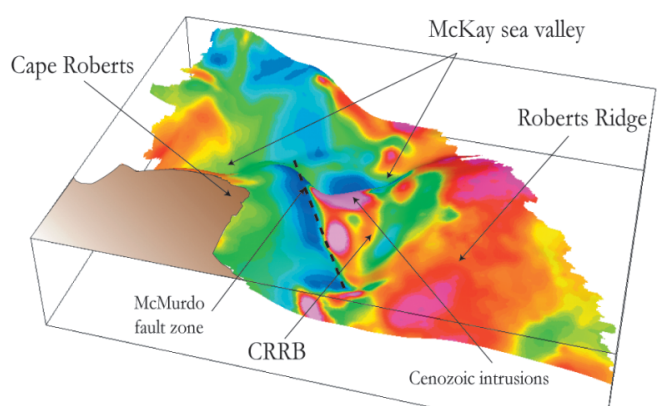

Fig. 3. Modal $3 \mathrm{D}$ view of CRRB with draped magnetic anomaly overprinted.

tains and the Ross Sea Rift (Ferraccioli and Bozzo, 2003) (fig. 2).

The CRRB HRAM (Cape Roberts Rift Basin High Resolution Aero Magnetic) survey was performed in the framework of the Cape Roberts Drilling Project (Davey et al., 2001). This was the first HRAM helicopter borne survey in Antarctica performed with a towed caesium magnetometer, sampling at $10 \mathrm{~Hz}$ with DGPS positioning (Bozzo et al., 1997). The survey was carried out in November 1994 during the GITARA IV (German Italian Aeromagnetic Research in Antarctica) campaign, at fixed altitude ( $150 \mathrm{~m}$ a.s.1.) with a line spacing of $500 \mathrm{~m}$, covering over $900 \mathrm{~km}^{2}$.
The CRRB (fig. 3) is a V-shaped trough about $500 \mathrm{~m}$ deep, interpreted as a graben (Armadillo et al., 2007), bounded to the east by a bathymetric high ( $100 \mathrm{~m}$ bsl) known as the Roberts Ridge (RR), and, to the west, by the foothills of the Transantarctic Mountains (TAM). The CRRB stratigraphy is provided both by seismic cruises (Behrendt et al., 1987; Hamilton et al., 2001) and three drillings (CRP1, CRP2, CRP3) located along the eastern flank of the graben. The graben is truncated to the north by the Pleistocene trough of the McKay sea valley, about $800 \mathrm{~m}$ deep.

The CRRB is filled with a complex sedimentary sequences ranging from latest Eocene to Miocene, which have been coded after Cooper (Cooper et al., 1987). Six seismic stratigraphic units (V1 - V5 sedimentary and V6 volcanic) and an acoustic basement (V7) are known in the CRRB-RR area (Davey et al., 2001). Magnetic susceptibility $\operatorname{logs}$ reports mean $k$ (magnetic susceptibility) values for the stratigraphic units filling the pull-apart basin (Sagnotti et al., 2001). Table I reports the $k$ values.

Table I. Mean $k$ values of CRP3 drill hole (fig. 3).

\begin{tabular}{cc}
\hline \hline Depth range [m b.s.f.] & Mean $k[10-5$ SI $]$ \\
\hline $0-243$ & 270 \\
$243-440$ & 132 \\
$440-627$ & 40 \\
$627-790$ & 223 \\
\hline
\end{tabular}

The acoustic basement is composed of Beacon supergroup sandstone with mean

$$
k=1 \div 10 \times 10^{-5} \mathrm{SI}
$$

The current shape and size of CRRB was inferred (Ferraccioli and Bozzo, 2003) relying on seismic data (Behrendt et al., 1987; Hamilton et al., 2001) combined with the aeromagnetic survey results.

The aeromagnetic data set was revised applying the modal decomposition proposed here. 

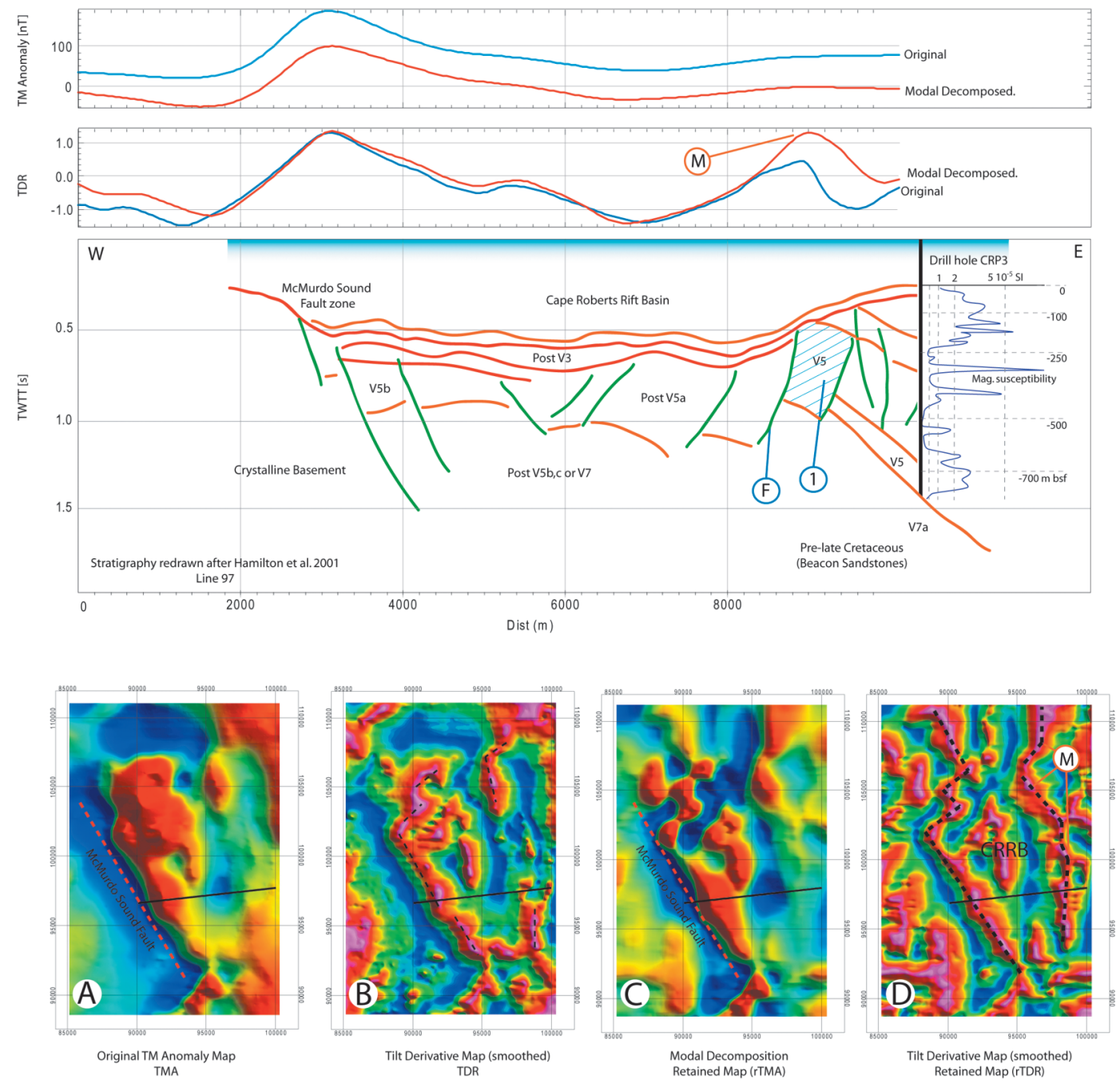

Fig. 4a-d. Modal decomposition CRRB HRAM. Above: TMI and tilt derivative profile data with a schematic geologic cross section (modified: Hamilton 2001). Below: a) Original TMI anomaly, b) Tilt derivatives calculated using the original data, c) Modal decomposition - «retained map» (rTMA), d) Tilt derivatives calculated using the «retained» dataset (rTDR).

The leveled and microleveled (Ferraccioli $e t$ $a l ., 1998)$ total magnetic intensity (TMI) anomaly draped map (Armadillo et al., 2007) was used as input for the proposed method.

The decomposition was performed using $\beta_{1}=3 ; \beta_{2}=14$.
The upper panel of fig. 4 shows the Total Magnetic Anomaly (TMA) and Tilt Derivatives (TDR) data (Verduzco et al., 2004) along a profile crossing the CRRB. The schematic geological cross section was redrawn after Hamilton $e t$ al. (2001). The TDR profile data calculated us- 


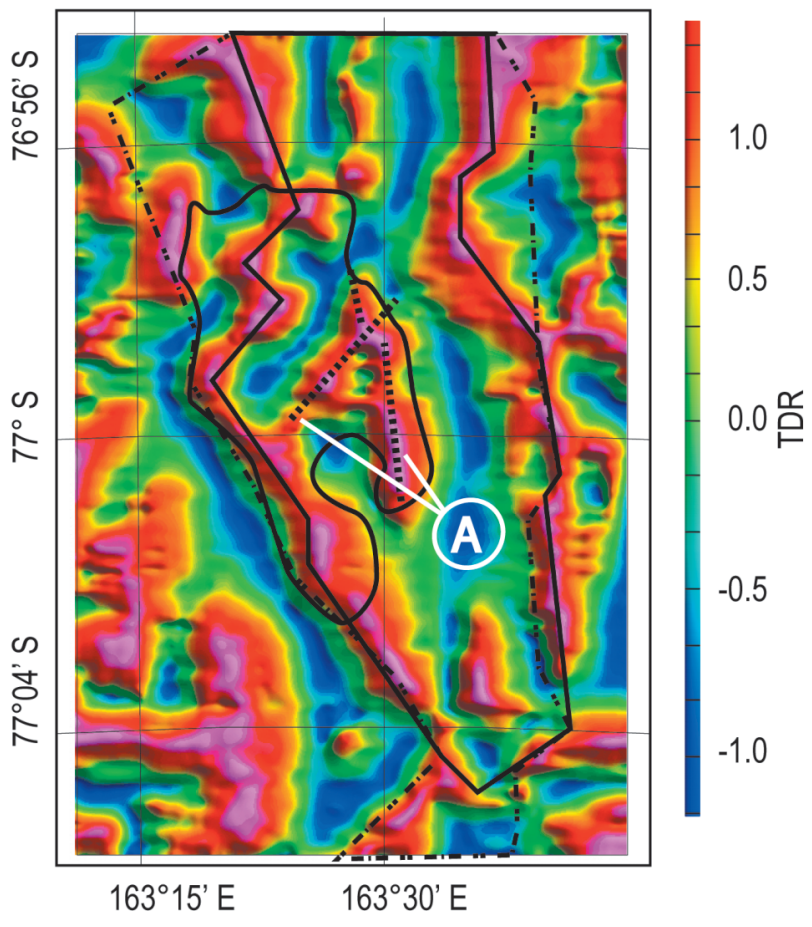

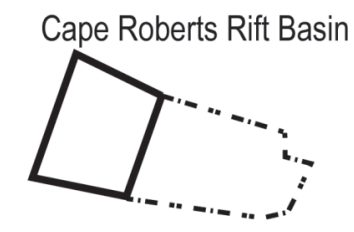

Outline of the main magnetic anomaly
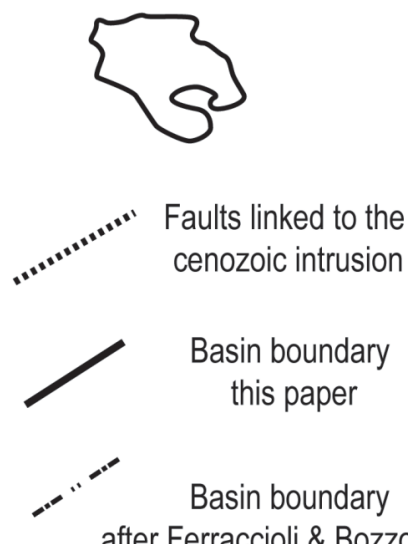

Basin boundary after Ferraccioli \& Bozzo 2003

Fig. 5. The Cape Roberts Rift Basin inferred using the Tilt derivatives map calculated using the «retained» dataset. Yellow dashed line: basin boundary (from Ferraccioli et al., 2003).

ing the «retained» dataset (red line) show a prominent maximum (M) corresponding to the right basin flank, not marked by the TMA map. The TDR algorithm is much more efficient if the calculation is applied to the modally decomposed dataset (retained dataset) instead of the original TMA.

The very low magnetic anomaly is presumably due to the uplift of block 1 (fig. 4) which places the V5 sediments, with interspersed thin high susceptibility layers (Ferrar Dolerites), closer to the sea bottom. Since the V5 mean susceptibility is not high (mean $k \sim 220 \times 10^{-5} \mathrm{SI}$ ), because of the small thickness of volcanic detrital layers, the absolute value of the magnetic anomaly and the magnetic lineament which marks the basin boundary (normal fault, fig. 4f) may not be visible using standard digital enhancement techniques.

The lower panel shows the original TMA map (A) and the TDR calculated using the original dataset (B). Box $\mathrm{C}$ show the modal decomposed (retained) map (rTMA, fig. 4d) and the TDR calculated using the «retained» dataset (rTDR). This representation exhibits the efficiency of the method.

The spatial persistence of a low amplitude feature ( $\mathrm{M}$ in fig. 4 lower panel) was singled out.

Figure 5 shows the interpretation of the CRRB boundary; the yellow dashed line reports the boundary proposed by Ferraccioli and Bozzo (2003) and the solid black line draws the tracing using the rTDR map.

The decomposition also shows a number of intra-basin faults (dotted lines, fig. 5a) identified as paths along which the known Cenozoic intrusions (Bozzo et al., 1997) took place. These lineaments were not visible from the original TMI anomaly map. 


\section{Conclusions}

It has been shown that the proposed method is efficient in removing noise and when used in synergy with TDR digital enhancement is able to reveal concealed features. In the example discussed the general shape of CRRB was confirmed and seismic lineaments matched with magnetic lineaments. A new set of magnetic lineaments was also highlighted. The proposed decomposition leads to magnetic anomaly maps that may significantly assist magnetic anomalies interpretation. The implementation of the filtering procedure is quick and the calculus, relying on both open source and commercial packages, requires very small resources. The empirical character of the filtering procedure requires a trial and error approach for a proper tuning of $\beta$ coefficients, making it convenient to implement the procedure as an inter- active tool. As a practical rule, the user can assume that the low amplitude noise is confined in the upper modes and the lower modes often carry broad features. The intermediate modes may hold subtle traits hidden by the regional and high amplitude features.

Keeping in mind the method's empirical nature, the results should be used to infer and verify the presence of magnetic lineaments.

\section{Acknowledgments}

The author is very grateful to E. Armadillo, A. Zunino (University of Genoa, Dip.Te.Ris.), D. Embriaco, M. Locritani (INGV) for their help and the useful discussions on this topic. The author also thanks prof. E. Bozzo of University of Genoa who provided the data used in this work.

\section{Appendix}

Here I provide a simple code (Octave/Matlab compatible) to perform the modal decomposition as illustrated above. The function called «Decomposition» needs a zero mean data matrix « $x$ » and the two thresholds b1, b2. $\mathrm{Xc}, \mathrm{Xt}, \mathrm{Xr}$ are the decomposed matrices, respectively the reconstructed, the retained and the residual dataset.

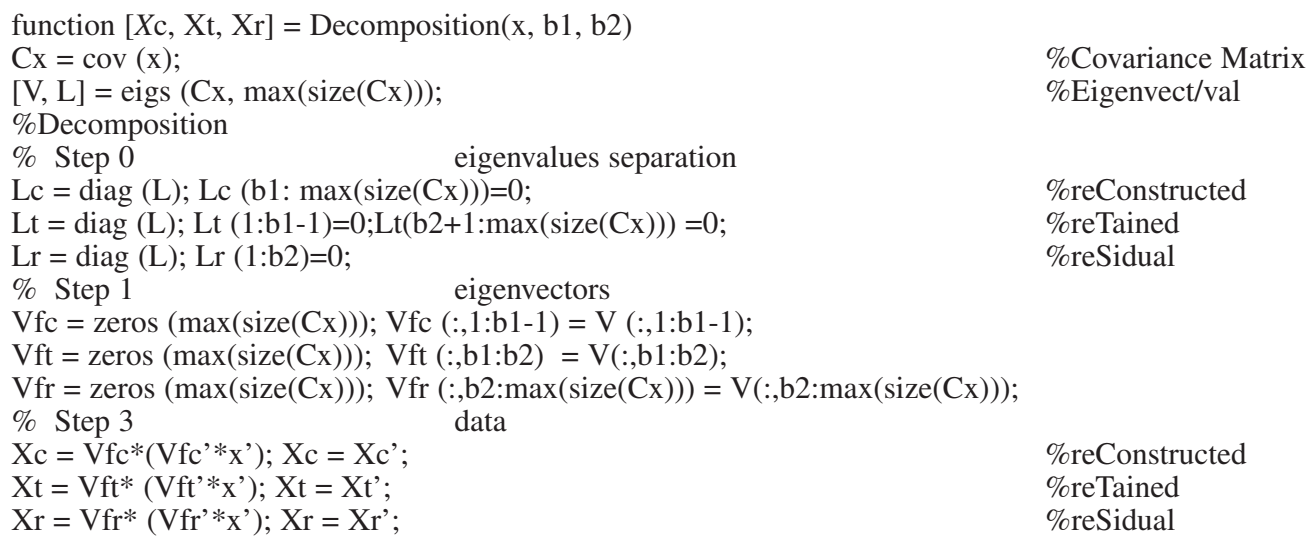

\%Covariance Matrix $\%$ Eigenvect/val

\%reConstructed

\%reTained

\%reSidual

\section{REFERENCES}

Armadillo, E., F. Ferraccioli, M. Gambetta, F. TalariCo, A. Zunino M. Zangani and E. Bozzo (2007): A high-resolution aeromagnetic survey over the Cape Roberts Rift Basin: Correlations with seismic reflection and magnetic susceptibility $\log$ data U.S. Geological Survey and The National Academies, USGS OF2007-1047, Extended Abstract, 076.
Behrendt, J.C., A.K. CoOper and A. YuAn (1987): Interpretation of marine magnetic gradiometer and multichannel seismic-reflection observations over the Western Ross Sea Shelf, Antarctica, in The Antarctic Continental Margin: Geology and Geophysics of the Western Ross Sea, edited by A.K. COOPER and F.J. DAvey, (Earth Science Series, 5B, Houston, Texas, CircumPacific Council for Energy and Mineral Resources), 155-177. 
Bozzo, E., D. Damaske, G. Caneva, M. Chiappini, F. FerRACCIOLI and M. GAMBETTA (1997): A high resolution aeromagnetic survey over proposed drill sites offshore of Cape Roberts in the Southwestern Ross Sea (Antarctica), in The Antarctic Region: Geological Evolution and Processes, edited by C.A. RICCI , 1129-1133.

Cooper, A.K., F.J. DAveY and J.C. BehrendT (1987): Seismic stratigraphy and structures in the Victoria Land Basin, estern Ross Sea, Antarctica., in The Antarctic continental margin: geology and geophysics of the western Ross Sea, edited by A.K. COOPER and F.J. DAVEY, (Circum-Pacific Res. Council, Houston), 27-76.

Davey, F.J., P.J. Barrett, M.B. Cita, F. Tessensohn, M. Thomson, K.J. WoOlfe, P.N. WebB and CAPE Roberts SCIENCE TEAM (2001): Drilling for Antarctic cenozoic climate and tectonic history at Cape Roberts, southwestern Ross Sea, EOS , 82 (585), 589-590.

EGBERT, G.D. and J.R. Booker (1989): Multivariate analysis of geomagnetic array data 1 , The response space Journal of Geophysical Research, 94 (B10), 1422714247.

Ferraccioli, F. and E. Bozzo (2003): Cenozoic strike-slip faulting from the eastern margin of the Wilkes Subglacial Basin to the western margin of the Ross Sea Rift: an aeromagnetic connection, (Geological Society, London), Special Publications, 210, 109-133, doi: 10.1144/GSL.SP.2003.210.01.07.

Ferraccioli, F., M. Gambetta and E. Bozzo (1998): Microlevelling procedures applied to regional aeromagnet- ic data : an example from the Transantarctic Mountains (Antarctica), Geophysical Prospecting, 46, 177-196.

FrEIRE, S.L. and T.J. UlRYCH (1988): Application of singular value decomposition to vertical seismic profiling, Geophysics, 53 (6), 778-785.

FUKUMORI, I., and C. WUNSCH (1991) Efficient representation of the North Atlantic hydrographic and chemical distribution, Progr.Ocean. , 27, 111-195.

Hamilton, R.J., B.P. LuYendyk, C.C. SoRlein and L.R. BARTEK (2001): Cenozoic tectonics of the Cape Roberts Rift Basin and Transantarctic Mountains Front, Southwestern Ross Sea, Antarctica Tectonics, 20 (3), 325-342.

Prasantha, H., H. Shashidhra and K.N. BalasubraMANYA MURTHY (2007): Image compression using SVD, Proceedings of the International Conference on Computational Intelligence and Multimedia Application, (ICCIMA 2007), 3, 143-145.

Sagnotti, L., K.L. Verosub, A.P. Roberts, F. Florindo and G.S. WILSON (2001): Environmental Magnetic Record of the Eocene-Oligocene Transition in CRP-3 Drillcore, Victoria Land Basin, Antarctica, Terra Antartica, 8 (4), 507-516.

Verduzco, B., D. Fairhead, C. Green and C. MacKenzie (2004): New insights into magnetic derivatives for structural mapping, The Leading Edge, 3, 116-120.

(received January 28, 2009; accepted April 20, 2009) 